\title{
Correction: Asthma and the risk of lung cancer: a meta-analysis
}

\section{Yan-Liang Qu, Jun Liu, Li-Xin Zhang, Chun-Min Wu, Ai-Jie Chu, Bao-Lei Wen, Chao Ma, Xu-yan Yan, Xin Zhang, De-Ming Wang, Xin Lv and Shu-Jian Hou}

Copyright: Qu et al. This is an open-access article distributed under the terms of the Creative Commons Attribution License 3.0 (CC BY 3.0), which permits unrestricted use, distribution, and reproduction in any medium, provided the original author and source are credited.

Present: The author's affiliations are listed incorrectly.

Correct: The proper affiliations are listed below.

\section{Yan-Liang $\mathbf{Q u}^{1, *}{ }^{1}$, Jun Liu ${ }^{2, *}$, Li-Xin Zhang ${ }^{1}$, Chun-Min Wu${ }^{1}$, Ai-Jie Chu ${ }^{1}$, Bao-Lei} Wen ${ }^{1}$, Chao Ma ${ }^{1}$, Xu-yan Yan ${ }^{1}$, Xin Zhang ${ }^{1}$, De-Ming Wang ${ }^{1}$, Xin Lv ${ }^{2}$, Shu-Jian Hou ${ }^{3}$

${ }^{1}$ Department of Anesthesiology, No. 401 Hospital of PLA, Qingdao 266071, Shandong, China

2 Department of Anesthesiology, Shanghai Pulmonary Hospital, Tongji University School of Medicine, Shanghai, China

${ }^{3}$ Department of Hand Surgery, No. 401 Hospital of PLA, Qingdao 266071, Shandong, China

Original article: Oncotarget. 2017; 8:11614-11620. https://doi.org/10.18632/oncotarget.14595 